\title{
Cholesterol-dependent hemolytic activity of Passiflora quadrangularis leaves
}

\section{L.N. Yuldasheva, E.B. Carvalho, M.-T.J.A. Catanho and O.V. Krasilnikov}

\begin{abstract}
Departamento de Biofísica e Radiobiologia, Centro de Ciências Biológicas,
\end{abstract} Universidade Federal de Pernambuco, Recife, PE, Brasil

\author{
Correspondence \\ O.V. Krasilnikov \\ Departamento de Biofísica \\ e Radiobiologia \\ CCB, UFPE \\ Av. Prof. Moraes Rego, s/n \\ 50670-901 Recife, PE \\ Brasil \\ Fax: +55-81-2126-8560 \\ E-mail: kras@ufpe.br \\ Research supported by CNPq. \\ $\ldots \ldots \ldots \ldots \ldots \ldots \ldots \ldots$
}

Received June 3, 2004

Accepted April 26, 2005

$\ldots \ldots \ldots \ldots \ldots \ldots \ldots$

\begin{abstract}
Plants used in traditional medicine are rich sources of hemolysins and cytolysins, which are potential bactericidal and anticancer drugs. The present study demonstrates for the first time the presence of a hemolysin in the leaves of Passiflora quadrangularis L. This hemolysin is heat stable, resistant to trypsin treatment, has the capacity to froth, and acts very rapidly. The hemolysin activity is dose-dependent, with a slope greater than 1 in a double-logarithmic plot. Polyethylene glycols of high molecular weight were able to reduce the rate of hemolysis, while liposomes containing cholesterol completely inhibited it. In contrast, liposomes containing phosphatidylcholine were ineffective. The Passiflora hemolysin markedly increased the conductance of planar lipid bilayers containing cholesterol but was ineffective in cholesterol-free bilayers. Successive extraction of the crude hemolysin with n-hexane, chloroform, ethyl acetate, and n-butanol resulted in a 10-fold purification, with the hemolytic activity being recovered in the n-butanol fraction. The data suggest that membrane cholesterol is the primary target for this hemolysin and that several hemolysin molecules form a large transmembrane water pore. The properties of the Passiflora hemolysin, such as its frothing ability, positive color reaction with vanillin, selective extraction with n-butanol, HPLC profile, cholesterol-dependent membrane susceptibility, formation of a stable complex with cholesterol, and rapid erythrocyte lysis kinetics indicate that it is probably a saponin.
\end{abstract}

\section{Introduction}

Pharmacological effects have been well established for many species of the genus Passiflora, collectively known in Brazil as "maracujá". Passiflora incarnata leaf extracts have a potent anxiolytic and sedative effect. Moreover, this plant is used in homeopathic medicine for the treatment of insomnia, epilepsy, tetanus, and muscle spasms (1). Hydroethanol extracts of $P$. alata and $P$.
Key words

- Passiflora quadrangularis

- Leaf extract

- Liposomes

- Bilayer permeation

- Hemolysin

- Membrane cholesterol edulis leaves presented anxiolytic activity (2). Passiflora quadrangularis has an antihelminthic action and is also frequently used to treat bronchitis, asthma, and whooping cough (3), and was even patented for treatment of diabetic complications and hypertension (4). Plants used in traditional folk medicine are a vast source of pharmacologically active components, including hemolysins and cytolysins, potential bactericidal and anticancer drugs (5-7). However, there 
are few publications about the cytotoxic activity of the family Passifloraceae (8-10).

Saponins are common constituents of plants that exhibit a broad spectrum of biological activities $(8,10-14)$, and frequently possess hemolytic, cytolytic and bactericidal activities $(6,8,15)$. Furthermore, saponins have plasma cholesterol-lowering activity $(5,12)$ and are widely utilized as a component of potent adjuvants to boost the immune response (16), principally when complexed with cholesterol (17). However, not all Passiflora species contain saponins in their leaves (18).

It is well known that saponins such as digitonin bind strongly to cholesterol to form a rigid equimolecular complex, and other saponins are also believed to interact with cholesterol $(19,20)$. Saponins usually increase membrane permeability (15) and this property is widely used to control permeabilization of cell membranes (21-23). In the case of erythrocytes, complex formation results in rapid cell lysis (24) and can lead to membrane disintegration in the presence of relatively large digitonin concentrations. The last effect suggests that digitonin has a detergent-like action. Digitonin forms so strong and stable a complex with cholesterol that it is used for cholesterol measurement in blood plasma, bile, and tissues. The purpose of the present study was to isolate a hemolytically active component from leaves of $P$. quadrangularis and to characterize its physical and biological properties.

\section{Material and Methods}

\section{Plant material}

P. quadrangularis leaves were collected in September 2001, from a garden in Carpina, PE, Brazil. The identity of the plant was confirmed by Dr. Marlene Barbosa, Botany Department, Federal University of Pernambuco. A voucher specimen of the plant was deposited in the IPA Herbarium (N56742) and was identical to the voucher specimen in the UFP (Recife) Herbarium (RG: 13616).

\section{Preparation of a crude extract containing saponins}

Fresh leaves of $P$. quadrangularis were mixed 1:2 (w/v) with deionized water and homogenized in a blender for about $5 \mathrm{~min}$. The homogenate was centrifuged at $3000 \mathrm{~g}$ for $10 \mathrm{~min}$ at $4^{\circ} \mathrm{C}$. The supernatant was collected and 10 volumes were mixed with one volume of absolute ethanol $(10: 1, \mathrm{v} / \mathrm{v})$ to stabilize the extract. This light yellow aqueous ethanol solution had relatively high osmolarity $(\sim 1,700 \mathrm{mOsm})$ and was acidic, $\mathrm{pH}$ 4.4. The concentration of solids in the supernatant was about $22.5 \mathrm{mg} / \mathrm{ml}$, as determined by drying to a constant weight at $60^{\circ} \mathrm{C}$.

Quantitative analysis of hemolytic activity requires careful control of the osmolarity and $\mathrm{pH}$ of all solutions to prevent swelling and nonspecific erythrocyte lysis. With this in mind, for the hemolytic assay, the primary aqueous ethanol solution was diluted 5.5 times with deionized water and the $\mathrm{pH}$ was adjusted to 7.4 with Tris base. The resulting slight sediment was removed by centrifugation at $3000 \mathrm{~g}$ for $5 \mathrm{~min}$. The final osmolarity of the diluted extract was 280 mOsm (Fiske Mark-3 Osmometer, Norwood, MA, USA). This adjusted extract possessed high hemolytic activity and will be referred to hereafter as crude hemolysin.

Partial purification of the active hemolysin from the crude hemolysin fraction was achieved by successive treatment of the crude hemolysin fraction with n-hexane, chloroform, ethyl acetate, and n-butanol. This method is frequently utilized for extracting saponins since the final $n$-butanol fraction is usually rich in saponins (18,25-27). All aqueous and organic extracts were tested for hemolytic activity. The solvents used in the organic extractions were first evaporated and the solid (gummy) residue of each extract 
was dissolved in an appropriate amount of a solution containing $150 \mathrm{mM} \mathrm{NaCl}$ and 5 $\mathrm{mM}$ Tris-citrate, $\mathrm{pH}$ 7.4. All solutions were adjusted to $280 \mathrm{mOsm}$ and to $\mathrm{pH}$ 7.4.

\section{Chemicals}

Egg yolk phosphatidylcholine (PC, type $\mathrm{V}-\mathrm{E})$ and cholesterol were purchased from Sigma (St. Louis, MO, USA). Trypsin was obtained from Boehringer (Ingelheim, Germany). Polyethylene glycols with average molecular masses of 6,000 and $35,000 \mathrm{kDa}$ were purchased from Fluka (Buchs, Switzerland). Sucrose, glucose, Tris base, citric acid, EDTA, ethanol, $\mathrm{NaCl}$, and Triton $\mathrm{X}$ 100 from various other suppliers were all of analytical grade. Milli-Q plus treated water (Millipore, Billerica, MA, USA) with resistivity of $18 \mathrm{M} \Omega-\mathrm{cm}$ was used to prepare all buffer solutions.

\section{Rabbit erythrocyte suspension}

For the hemolysis assay, rabbit erythrocytes were used as described previously (28). Briefly, fresh blood was mixed with 20 volumes of isotonic standard physiological solution (SPS, $150 \mathrm{mM} \mathrm{NaCl}, 5 \mathrm{mM}$ Triscitrate, $1 \%$ EDTA, pH 7.4). Rabbit erythrocytes were obtained by centrifugation at 1000 $g$ for $5 \mathrm{~min}$. The supernatant was discarded and sedimented erythrocytes were then washed three times with the same buffer, centrifuged and resuspended in EDTA-free SPS. The hematocrit of the final suspension was measured with a capillary hematocrit centrifuge (model 210 I.E.C., FANEM, São Paulo, SP, Brazil) and adjusted to a $2 \%(w /$ v) suspension, which was used throughout the study.

\section{Liposome preparation}

Lipids (20 mg PC, or a mixture of $10 \mathrm{mg}$ PC with $10 \mathrm{mg}$ cholesterol) were dissolved in Folch solution (chloroform:methanol, 2:1, $\mathrm{v} / \mathrm{v})$, transferred to a round-bottom glass flask and dried with a nitrogen stream to form a thin film. To make the liposomes, 1 $\mathrm{ml}$ SPS was added to the flask and vigorously shaken to remove the lipid film from the flask wall. Finally the mixture was sonicated (Mini-som, Thornton INPEC Eletrónica, Vinhedo, São Paulo, SP, Brazil) to yield an aqueous vesicle suspension. The lipid concentration of this liposome suspension was $20 \mathrm{mg} / \mathrm{ml}$.

\section{Hemolytic assay}

Hemolysis was assayed at $25 \pm 2^{\circ} \mathrm{C}$ using a Bio-Rad plate reader (model 170-6638, with replacement lamp\#3550: Hercules, CA, USA). The extent of lysis was quantified by direct measurement of cell suspension absorbance at $655 \mathrm{~nm}$. At this wavelength hemolysis causes a decrease in absorbance. All substances were dissolved in SPS. Extracts were serially double diluted with SPS (which in other experiments was complemented with non-electrolytes or liposomes at the desired concentrations). The process was initiated by the addition of a $2 \%$ suspension of rabbit erythrocytes. Absorbance was repeatedly measured in all wells at appropriate time intervals (usually $1-5 \mathrm{~min}$ ). The final concentration of erythrocytes was $1 \%$ and the final volume in each microplate well was $100-200 \mu l$. Wells containing only a $1 \%$ erythrocyte suspension in SPS were used for a negative control assay ( $0 \%$ hemolysis). Absorbance of wells with erythrocytes lysed with $2 \%$ Triton X-100 was taken as $100 \%$ hemolysis. The percentage of hemolysis in other wells was calculated relative to the Triton X-100 value. A multichannel pipette was used for simultaneous initiation of the assay and the first absorbance reading was taken immediately.

\section{Hemolysis/K+ $\mathbf{K}^{+}$-efflux assay}

In this assay the kinetics of erythrocyte 
lysis and the estimation of $\mathrm{K}^{+}$-efflux from erythrocytes were measured in parallel in experiments carried out in glass tubes at $37 \pm$ $1^{\circ} \mathrm{C}$ in a final volume of $10 \mathrm{ml}$. At appropriate times a 1-ml aliquot of the erythrocyte suspension was centrifuged in Eppendorf tubes $(30 \mathrm{~s}$ at $1000 \mathrm{~g}$ ). The extent of lysis was estimated by determining the absorbance of the supernatant at $540 \mathrm{~nm}$. The $\mathrm{K}^{+}$ concentration in the supernatant was measured with a $\mathrm{K}^{+}$-selective electrode. All other conditions were as described for the hemolysis assay.

\section{Transmembrane current assay}

Planar bilayer lipid membranes (BLMs) were used to measure the change in membrane conductance in the presence of hemolysin. BLMs were formed at $25^{\circ} \mathrm{C}$ by the technique of Mueller et al. (29) in an Ussing Teflon chamber, whose two compartments were separated by a $20-\mu \mathrm{m}$ Teflon diaphragm with an orifice for bilayer formation $\sim 300$ $\mu \mathrm{m}$ in diameter. Bilayers were formed in the orifice of the chamber by applying a drop of $2 \%$ lipid solution in n-decane. Membrane formation was monitored using a binocular microscope and by observing a marked increase in capacitance. The electrical characteristics of BLMs were measured under voltage clamp conditions as previously described (28). The amplifier signal was monitored with a Nicolet-2090-III storage oscilloscope (Nicolet Technologies, Madison, WI, USA) and recorded on an IBM-compatible 486/ $487100 \mathrm{MHz}$ computer with a DT01-EZ 12 bit A/D converter board (Data Translation, Marlboro, MA, USA). Whole Cell Electrophysiology software (WCP V1.7b) developed by Dr. J. Dempster (University of Strathclyde, Glasgow, Scotland, UK) was employed for data analysis.

The trans-compartment of the experimental chamber was connected to the virtual ground and voltage pulses $(40 \mathrm{mV})$ were applied to the cis-compartment of the cham- ber, to which the hemolysin was also added. The conductance of bilayer membranes $(G)$ in symmetrical solutions was defined as $G=I / V$, where $I$ is the transmembrane current flowing through the membrane and $V$, corresponds to the fixed potential. The basal conductance of BLMs was less than $5 \mathrm{pS}$.

\section{Testing the properties of the hemolysin}

To study the properties of the hemolysin and to clarify its target at the membrane level, hemolysin was pre-incubated with trypsin or with liposomes (prepared from PC or from a $\mathrm{PC} /$ cholesterol mixture) for $3 \mathrm{~h}$ in a water bath at $37^{\circ} \mathrm{C}$. The final concentrations of hemolysin, lipids and trypsin were 2, 2 and $0.02 \mathrm{mg} / \mathrm{ml}$, respectively. The hemolytic activity of these pre-treated samples was tested as described in "Hemolytic assay".

In order to determine the stoichiometry of the hemolysin/cholesterol interaction, a constant amount of the hemolysin $(0.1 \mathrm{mg})$ was mixed with different amounts of cholesterol-containing liposomes directly in 96well plates just before the addition of the erythrocyte suspension.

The osmotic balance method (28) was used in an attempt to estimate the size of pores induced by hemolysin in erythrocyte membranes. Non-electrolytes of different sizes (glucose, sucrose and polyethylene glycols) were added to SPS at concentrations that increased the osmolarity of the solution by $40 \mathrm{mOsm}$ (this increment is close to the osmotic pressure created by intracellular hemoglobin). This method assumes that hemolysis does not occur when the size of waterfilled pores induced by any hemolysin in an erythrocyte membrane is smaller than the hydrodynamic size of the non-electrolyte molecules added to SPS.

To assess the thermal stability of the hemolysin, a sample was pre-heated at $100^{\circ} \mathrm{C}$ for $10 \mathrm{~min}$ and its hemolytic activity was 
then evaluated by the normal procedure. A Fiske Mark-3 Osmometer was used to measure the osmolarity of all solutions used.

\section{Statistical analysis}

The Student $t$-test was used to evaluate the significance of the difference between mean values. Data are reported as means \pm SD.

\section{Results}

Temperature and trypsin sensitivity of the $P$. quadrangularis hemolytic activity

The hydroethanol solution of $P$. quadrangularis leaves possessed high lytic potency against erythrocytes. The $\mathrm{K}^{+}$efflux always preceded hemolysis (data not shown), indicating that hemolysis may have been of an osmotic nature. Hemolytic activity increased after $3 \mathrm{~h}$ of trypsin treatment (Figure $1)$. The lysis half-time of a $1 \%$ erythrocyte suspension $\left(\mathrm{T}_{50 \%}\right)$ in the presence of $30 \mu \mathrm{g} /$ $\mathrm{ml}$ hemolysin was $2.5 \pm 0.1 \mathrm{~min}$. The trypsin treatment decreased $\mathrm{T}_{50 \%}$ to $1.5 \pm 0.1 \mathrm{~min}$. It seems that the hemolysin is partially associated with proteins and trypsin digestion releases it from a protein-hemolysin complex. The hemolytic activity of the hemolysin was practically unaltered by 10 min heating at $100^{\circ} \mathrm{C}$ (data not shown). The hemolysin was positive in the froth test.

The foregoing results indicate that the hemolysin is not a protein because it does not lose activity after heating and protease treatment. The pre-lytic $\mathrm{K}^{+}$efflux suggests the osmotic nature of hemolysis and provides evidence of pore formation in erythrocyte membranes. Once formed, transmembrane water pores apparently provoke a cascade of events including water entry, erythrocyte swelling and membrane rupture, measured as hemoglobin release. Since pore size is a key parameter, experiments were carried out to estimate it.

\section{Pore-sizing experiments}

The osmotic balance method was used to evaluate the radius of water pores induced by the hemolysin. It was found that small nonelectrolytes have no influence on hemolysin activity. On the other hand, large non-electrolytes (especially PEG 35000) were able to decelerate the hemolysis, but were not able to prevent it completely (Figure 2 ). The combination of pre-lytic $\mathrm{K}^{+}$-efflux with the absence of complete blockage of hemolysis by large non-electrolytes such as PEG 35000 suggests that pores formed by hemolysin in the membrane are heterogeneous. Pore size heterogeneity has been reported for other lipophilic hemolysins in erythrocyte membranes (30). However, we cannot exclude the possibility that the hemolysin pores may simply be larger than the hydrodynamic radius of the largest non-electrolyte (PEG $35000=$ $\sim 3.7 \mathrm{~nm}$ ) used in our study.
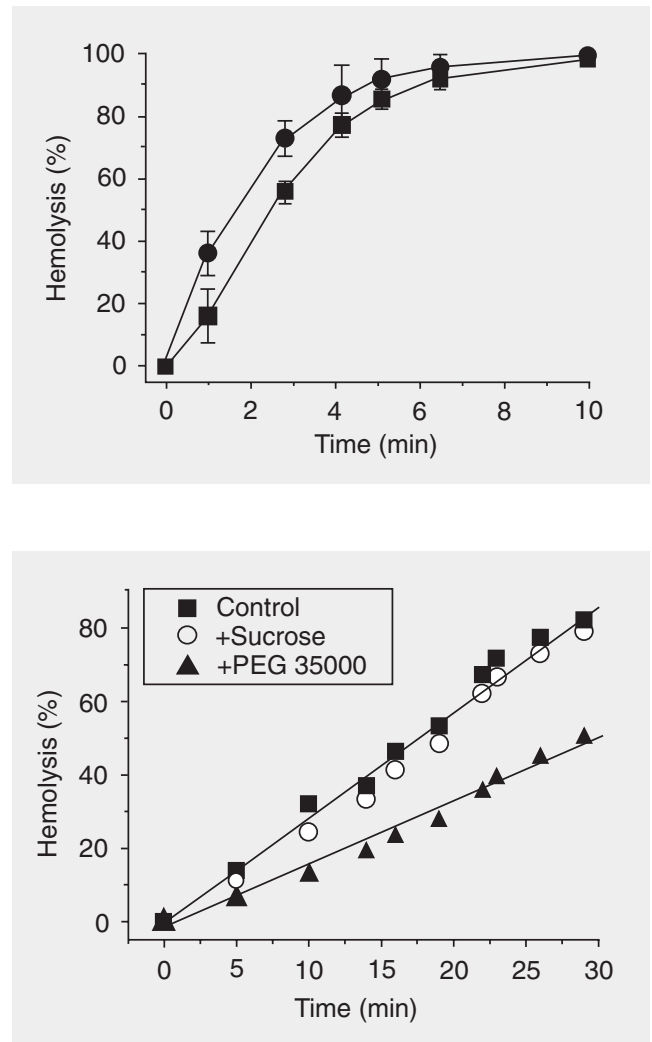

Figure 1. Time course of hemolysis in response to the addition of $30 \mu \mathrm{g} / \mathrm{ml}$ Passiflora quadrangularis hemolysin. Squares = original preparation of hemolysin, circles $=$ hemolysin pre treated with trypsin. Data are reported as the mean \pm SD for at least 4 experiments. The time requirement for $50 \%$ erythrocyte lysis $\left(T_{50 \%}\right)$ was equal to $2.5 \pm$ $0.1 \mathrm{~min}$ and $1.5 \pm 0.1 \mathrm{~min}$ for the original preparation of hemoly$\sin$ and for the trypsin-treated one, respectively. All other conditions are described in Material and Methods.

Figure 2. Influence of non-electrolytes on the hemolysin activity. The final concentration of hemolysin was $15 \mu \mathrm{g} / \mathrm{ml}$. Results of typical experiments are presented. Other non-electrolytes tested (glucose, maltose and low molecule mass polyethylene glycols (PEGs, up to 1000)) had the same negligible effect as sucrose. All other conditions are described in Material and Methods. 
Figure 3. Concentration-dependent hemolysis by Passiflora molysis was quantified following incubation for $1 \mathrm{~min}$. Data are reported as mean \pm SEM percent hemolysis for 4 experiments in a double logarithmic plot. The SEM was $<3 \%$. Straight lines are least-squares fit. The slope value was $1.3 \pm$ 0.1 . All other conditions for the experiment are described in Material and Methods. quadrangularis hemolysin. He-

Dose dependence and estimation of the number of hemolysin molecules forming an elementary pore

To determine how many molecules of hemolysin comprise the transmembrane water pore, the dependence of erythrocyte

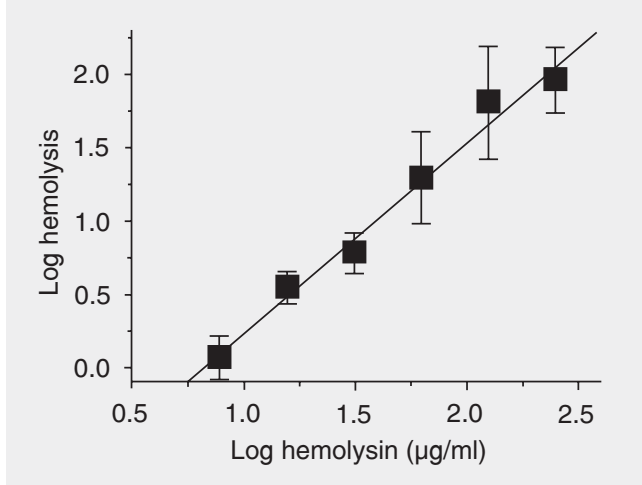

Figure 4. Influence of liposomes on the hemolytic activity of the Passiflora quadrangularis hemolysin. The hemolysin was preincubated with liposomes as described in Material and Methods. The hemolysin samples were serially double diluted with standard physiological solution and the erythrocyte suspension was then added. Hemolysis was quantified following incubation for $1 \mathrm{~min}$. Data are reported as mean \pm SD percent hemolysis for 4 experiments. Squares, tri-

angles, and inverted triangles show the effects of untreated hemolysin, and of hemolysin pre-incubated with phosphatidylcholine (PC) liposomes and PC/cholesterol (Chol) liposomes, respectively. All other conditions for the experiment are described in Material and Methods.

Figure 5. Typical recordings of transmembrane current under the influence of the hemolysin. Hemolysin (final concentration: $20 \mu \mathrm{g} / \mathrm{ml}$ ) was added to the ciscompartment at the time indicated by the arrow. Planar bilayers were formed from pure phosphatidylcholine $(P C)(A)$ or from a $\mathrm{PC} /$ cholesterol mixture $(1: 1 ; w / w)(B)$ by the method of Mueller et al. (29). Current and time scales are given in the figure. Other conditions are described in Material and Methods. lysis on hemolysin concentration was investigated. The slope of the dose dependence in a double-logarithmic plot was found to be $>1$ (Figure 3). As shown elsewhere (30,31), it suggests that more than one molecule of hemolysin participates in the formation of an elementary permeabilizing unit in erythrocyte membrane.

\section{Membrane cholesterol as a target for the action of hemolysin}

In order to identify the membrane component responsible for cell sensitivity to the hemolysin, liposomes of different lipid compositions were used. PC liposomes added to the erythrocyte suspension did not prevent hemolysis, but those containing cholesterol were able to completely abolish it (Figure 4).

To clarify the role of membrane cholesterol in hemolysis, additional experiments with planar lipid bilayer membranes were carried out. We found that PC-BLMs were practically insensitive to the hemolysin, whereas the conductance of cholesterol-containing bilayers increased strongly in the presence of hemolysin in bath solutions (Figure 5).

To estimate the concentration of the hemolytically active component in our hemolysin preparation, serial 2-fold dilutions of cholesterol-containing liposomes were supplemented with a fixed amount (equal to 0.1 mg dry weight) of the hemolysin. The reaction was started by erythrocyte addition and the extent of hemolysis was measured at 1 , 10, and $60 \mathrm{~min}$ (Figure 6). Fifty percent inhibition of hemolysis occurred only when the dry weight of hemolysin exceeded the weight of cholesterol by 5 -fold. The hemolysin-cholesterol complex appeared to be stable, with no conspicuous release of hemolysin from this complex for at least $60 \mathrm{~min}$ (data not shown).

Several lines of evidence suggest that the P. quadrangularis hemolysin is a saponin. A 1:1 molar ratio of cholesterol/hemolysin was 
sufficient to prevent hemolysis, as was established for digitonin $(19,20)$. The molecular weight of saponin is comparable to that of cholesterol, which is accessible to the hemolysin only in the external leaflet of liposome membranes. On this basis, one can estimate that the crude hemolysin preparation may contain up to $10 \%$ (by weight) of the active hemolysin. Hence, dry P. quadrangularis leaves may contain up to $0.8 \%$ active component. It is possible that this approach overestimates the hemolysin content. The actual value could be still lower if a small percentage of multilayer liposomes were present.

\section{Selective saponin extraction and the $P$. quadrangularis hemolysin}

To further explore the possibility that the $P$. quadrangularis hemolysin is a saponin, the traditional selective saponin extraction procedure was used. Four organic solvents (n-hexane, chloroform, ethyl acetate, and nbutanol) were successively employed. With this method, the last n-butanol fraction contains crude saponin. The highest specific hemolytic activity $(660 \mathrm{HU} / \mathrm{mg})$ was found in the n-butanol extract (Table 1). This value is $~ 10$ times higher than those in the initial hydroethanol solution or in any other solutions/phases in the purification process. Experiments analogous to those in Figures 1-6 were done with this partially purified hemolysin (n-butanol fraction). The data obtained were identical except for the hemolysin concentration, which was $\sim 10$ times less (data not shown). Moreover, the acid hydrolysis products of this hemolysin gave a color reaction with vanillin, a classic test for saponins (32).

\section{Discussion}

The present study demonstrates for the first time that leaves of $P$. quadrangularis possess a potent, heat-stable, non-proteinaceous hemolysin(s) and that its activity is increased by trypsin treatment. This activa-

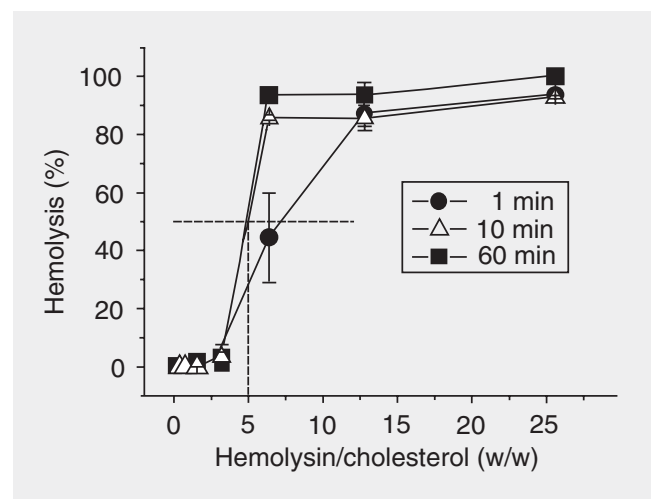

Figure 6. Titration of the hemolytic effect of the Passiflora quadrangularis hemolysin with cholesterol. Phosphatidylcholine/cholesterol liposomes were two-fold serially diluted on microplates and mixed with a fixed amount $(0.1 \mathrm{mg}$ dry weight) of the hemolysin and the erythrocyte suspension was then added. Hemolysis was quantified following incubation for the time given in figure. The hemolysin/cholesterol ratio $(\mathrm{w} / \mathrm{w})$ is given on the abscissa. The arrow indicates a condition in which $50 \%$ inhibition of hemolysis was observed. Data are reported as mean \pm SD percent hemolysis for 4 experiments. Circles $=1 \mathrm{~min}$; triangles $=10$ $\mathrm{min}$; squares $=60 \mathrm{~min}$.

Table 1. Distribution of hemolytic activity among the fractions used for saponin extraction.

\begin{tabular}{lrcr}
\hline & $\begin{array}{c}\text { Dry weight } \\
\text { (mg) }\end{array}$ & $\begin{array}{c}\text { Specific } \\
\text { activity } \\
\text { (units/mg) }\end{array}$ & $\begin{array}{r}\text { Total } \\
\text { activity } \\
\text { (units) }\end{array}$ \\
\hline $\begin{array}{l}\text { Hydroethanol } \\
\text { extract }\end{array}$ & 5400 & 64 & 345,600 \\
n-Hexane & 1500 & 10 & 15,000 \\
Water ${ }^{\mathrm{H}}$ phase & 3430 & 80 & 274,400 \\
Chloroform & 700 & 20 & 14,000 \\
Water $\mathrm{C}$ phase & 2100 & 80 & 168,000 \\
Ethyl acetate & 420 & 20 & 8,400 \\
Water $\mathrm{E}$ phase & 1480 & 80 & 118,400 \\
n-Butanol & 155 & 660 & 102,300 \\
Water ${ }^{\mathrm{B}}$ phase & 1360 & 10 & 13,600 \\
\hline
\end{tabular}

One hemolytic unit is the amount of hemolysin that is able to completely lyse $100 \mu$ of a $1 \%$ erythrocyte suspension in $5 \mathrm{~min}$ at $25 \pm 1^{\circ} \mathrm{C}$. Loss in total activity seen at each step of the purification procedure is the result of discarding the interphase zone material. Each value is the mean of three replicates with a standard deviation of not more than $20 \%$ of the mean.

tion or "unmasking" is a common feature of biologically active plant components such as saponins, many of which are present in the plant in inactive states that can be converted to active forms in situ or in vitro (5).

The kinetics of erythrocyte lysis was fast. The hemolysin-induced pores in erythrocyte membranes were large and probably heterogeneous. It is likely that several hemolysin 
molecules participate in transmembrane pore formation. In an attempt to show the level of oligomerization achieved by the hemolysin during pore formation we analyzed the doseeffect relationship. We established that the slope of the dose dependence in doublelogarithmic plots exceeded one. This method has been widely employed for establishing the number of molecules necessary for the formation of a pore in erythrocyte membranes and in planar lipid bilayers by different types of membrane active components $(30,31,33)$. From the slope we conclude that more than one molecule of the hemolysin participates in the formation of an elementary pore in erythrocyte membranes.

To determine the nature of the membrane target responsible for the high sensitivity of erythrocyte membranes to the hemolysin, we employed lipid bilayers (spherical and planar). By using liposomes of different lipid compositions we found that PC-liposomes did not prevent hemolysis. On the other hand, the ability of cholesterol-containing liposomes to prevent erythrocyte lysis, even in the presence of large hemolysin concentrations, was impressive. The most plausible explanation would be the preferential binding of the hemolysin to cholesterol-containing liposomes, which protect erythrocytes from hemolysis. The difference between control hemolysin activity and activity in the presence of PC-liposomes was not statistically reliable at all hemolysin con-

Figure 7. HPLC chromatogram of Passiflora quadrangularis hemolysin. HPLC conditions: C18Waters column, $3.8 \mathrm{~mm}$ internal diameter x $300 \mathrm{~mm}, 15-20$ $\mu \mathrm{m}$ operated at room temperature $\left(22^{\circ} \mathrm{C}\right)$ with a mobile phase of $\mathrm{CH}_{3} \mathrm{OH} / \mathrm{H}_{2} \mathrm{O}=50 / 50$ at a flow rate of $1 \mathrm{ml} / \mathrm{min}$. The effluent was monitored at $215 \mathrm{~nm}$ using an AKTA $^{\mathrm{TM}}$ chromatography system (GE-Amersham Biosciences, Piscataway, NJ, USA). The approximate elution position of the hemolysin is indicated by the solid horizontal line. terol is the probable target for the hemolysin. Hemolysin-cholesterol complexes seem to be very stable, since we did not observe any release of hemolysin from this complex during a 60-min period.

Experiments with planar lipid bilayer membranes gave additional support to this apparent role of cholesterol. We established that BLMs formed from pure PC were insensitive to the hemolysin, whereas the conductance of cholesterol-containing bilayers was significantly increased by the hemolysin in a time-dependent manner. These results indicate that cholesterol is a target for the action of this hemolysin against membranes.

In addition, characteristics such as resistance to heating and to trypsin treatment, specificity for and formation of a stable complex with cholesterol, as well as frothing ability and a positive vanillin color test, all suggest that the hemolysin is indeed a saponin.

In order to confirm this point, the hemolysin was extracted by a procedure developed for saponins, which involves successive extractions of the crude hemolysin with n-hexane, chloroform, ethyl acetate, and n-butanol. A 10-fold purification was achieved. The hemolytic activity, as expected $(18,25-27)$, was recovered in the butanol fraction. Moreover, when subjected to HPLC analysis (Figure 7), the hemolysin's profile resembled those of other saponin-containing samples chromatographed under similar conditions (34), thus confirming its saponin nature.

Although saponins possess detergent-like properties (e.g., positive froth test) due to their amphiphilic character, and detergents are able to form pores in membranes (35), our data suggest that the $P$. quadrangularis hemolysin does not induce membrane disorder through a simple detergent action. It is well known $(36,37)$ that membrane destabilization by detergents is mostly influenced by the membrane physical state, bilayers in the gel state being much more resistant to 
membrane-disruptive agents than bilayers in the liquid-crystalline state. Cholesterol enhances membrane resistance to detergents. In the present study, only bilayers containing cholesterol were sensitive to the action of the hemolysin. Moreover, for a series of saponins (digitonin, aescine, tomatine, stevioside, and ginsenoside Rg1) it was demonstrated (38) that neither the surface properties nor the interfacial tension-lowering properties of saponins (in n-decane/water) could be correlated with their ability to induce hemolysis.

Our results showing that only cholesterol-containing BLMs are susceptible to the hemolysin are reminiscent of earlier studies (39) in which it was reported that digitonin induced a noticeable change in glucose permeability of vesicles of egg yolk lecithin containing cholesterol, but not of cholesterol-free vesicles. Digitonin/cholesterol complex formation in the membranes has been established a long time ago $(19,20)$, and these data suggest that complex formation might be related to the hemolytic activity. Moreover, although saponins possess detergent-like properties, they can increase the permeability of cell membranes without destroying them, and therefore are used for the detection of intracellular antigens on intact cells $(22,23)$. Consistent with these studies, we propose that the $P$. quadrangularis hemolysin also acts by forming a com- plex with cholesterol that creates transmembrane pores in erythrocyte membranes, resulting in water influx and finally leading to swelling and membrane rupture.

The name 'saponin' denotes a large and chemically heterogeneous group of sterol glycosides and triterpene glycosides linked to one or two polar oligosaccharides. The rates of hemolysis induced by sterol glycosides are generally much higher than those of triterpene glycosides (24). The high rate of erythrocyte lysis evoked by the $P$. quadrangularis hemolysin and its prevention by cholesterol-containing liposomes suggest that this hemolysin may belong to the sterol glycoside group. Its exact chemical structure will be the subject of further study.

\section{Acknowledgments}

We are indebted to Dr. Yara de Miranda Gomes (Centro de Pesquisas Aggeu Magalhães, FioCruz) for the use of a microplate reader and to Dr. José Luiz de Lima Filho (Laboratório de Imunopatologia Keizo Asami, LIKA, Federal University of Pernambuco, Recife, PE, Brazil) for allowing us to use the $\mathrm{AKTA}^{\mathrm{TM}}$ purifier. We are grateful to Dr. Steven D. Aird (Department of Biology, Norfolk State University, Norfolk, VA, USA) for editing, that improved the clarity of the manuscript.

\section{References}

1. Dhawan K, Kumar S \& Sharma A (2001). Comparative biological activity study on Passiflora incarnata and $P$. edulis. Fitoterapia, 72: 698-702.

2. Petry RD, Reginatto F, de-Paris $F$ et al. (2001). Comparative pharmacological study of hydroethanol extracts of Passiflora alata and Passiflora edulis leaves. Phytotherapy Research, 2: 162-164.

3. Mowrey DB (1993). Herbal Tonic Therapies. Keats Publishing, New Canaan, CT, USA.

4. Nippon MKK (1993). Angiotensin converting enzyme and aldosereductase inhibitory agent - comprises Passiflora quadrangularis extract with organic solvent or water, or vitexin. Patent No. 1995009562 [02].

5. Chandel RS \& Rastogi RP (1980). Triterpenoid saponins and sapogenins: 1973-1978. Phytochemistry, 19: 1889-1908.
6. Rao AV \& Sung MK (1995). Saponins as anticarcinogens. Journal of Nutrition, 125 (Suppl): 717S-724S.

7. Shao Y, Chin CK, Ho CT et al. (1996). Anti-tumor activity of the crude saponins obtained from asparagus. Cancer Letters, 104: 3136.

8. Birner J \& Nicolls JM (1973). Passicol, an antibacterial and antifungal agent produced by Passiflora plant species: preparation and physicochemical characteristics. Antimicrobial Agents and Chemotherapy, 3: 105-109.

9. Lutomski J \& Malek B (1975). Pharmacochemical investigations on raw materials of the genus Passiflora. 3. Phytochemical investigations on raw materials of Passiflora edulis forma Flavicarpa. Planta Medica, 27: 222-225.

10. Perry NB, Albertson GD, Blunt JW et al. (1991). 4.4-Hydroxy-2- 
cyclopentenone an antipseudomonas and cytotoxic component from Passiflora-tetrandra. Planta Medica, 57: 129-131.

11. Rao AV \& Gurfinkel DM (2000). The bioactivity of saponins: triterpenoid and steroidal glycosides. Drug Metabolism and Drug Interactions, 17: 211-235.

12. Kinlen PJ, Menon VP \& Pirakitikulr V (2001). Composition useful as a food additive for reducing serum cholesterol levels comprises a mixture of a phytostanol or its ester and a surfactant (Patent number: WO200132036-A1; AU200114636-A). International Patent Classification: A23L-001/035; BNA2-3L001/30.

13. Ridoux O, Di Giorgio C, Delmas F et al. (2001). In vitro antileishmanial activity of three saponins isolated from ivy, alpha-hederin, betahederin and hederacolchiside $A_{1}$ in association with pentamidine and amphotericin B. Phytotherapy Research, 15: 298-301.

14. Feng $X Z$, Dong $M$ \& Xu SX (2001). A new triterpenoidal saponin from Ixeris sonchifolia and its cytotoxic activity. Pharmazie, 56: 663664.

15. Li XX, Davis B, Haridas V et al. (2005). Proapoptotic triterpene electrophiles (avicins) form channels in membranes: cholesterol dependence. Biophysical Journal, 88: 2577-2584.

16. Santos WR, Bernardo RR, Peçanha LM et al. (1997). Haemolytic activities of plant saponins and adjuvants. Effects of Periandra mediterranea saponin on the humoral response to the FML antigen of Leishmania donovani. Vaccine, 15: 1024-1029.

17. Kersten GF, Spiekstra A, Beuvery EC et al. (1991). On the structure of immune-stimulated saponin-lipid complexes (iscoms). Biochimica et Biophysica Acta, 1062: 165-171.

18. Reginatto FH, Kauffmann C, Schripsema J et al. (2001). Steroidal and triterpenoidal glucosides from Passiflora alata. Journal of the Brazilian Chemical Society, 12: 32-36.

19. Akiyama T, Takagi S, Sankawa U et al. (1980). Saponin-cholesterol interaction in the multibilayers of egg yolk lecithin as studied by deuterium nuclear magnetic resonance: digitonin and its analogues. Biochemistry, 19: 1904-1911.

20. Nishikawa M, Nojima S, Akiyama T et al. (1984). Interaction of digitonin and its analogs with membrane cholesterol. Journal of Biochemistry, 96: 1231-1239.

21. Lapetina EG, Watson SP \& Cuatrecasas P (1984). Myo-inositol 1,4,5-trisphosphate stimulates protein phosphorylation in saponinpermeabilized human platelets. Proceedings of the National Academy of Sciences, USA, 81: 7431-7435.

22. Goldenthal KL, Hedman K, Chen JW et al. (1985). Postfixation detergent treatment for immunofluorescence suppresses localization of some integral membrane proteins. Journal of Histochemistry and Cytochemistry, 33: 813-820.

23. Jacob MC, Favre M \& Bensa JC (1991). Membrane cell permeabilization with saponin and multiparametric analysis by flow cytometry. Cytometry, 12: 550-558.

24. Takechi M \& Tanaka $Y$ (1995). Haemolytic time course differences between steroid and triterpenoid saponins. Planta Medica, 61: 7677.

25. Sotheeswaran S, Bokel M \& Kraus W (1989). A hemolytic saponin, randianin, from Randia Dumetorum. Phytochemistry, 28: 1544-1546.

26. Mimaki Y, Kanmoto T, Sashida $Y$ et al. (1996). Steroidal saponins from the underground parts of Chlorophytum comosum and their inhibitory activity on tumor promoter-induced phospholipids metabolism of HELA cells. Phytochemistry, 41: 1405-1410.

27. Itabashi M, Segawa K, Ikeda $Y$ et al. (2000). A new bioactive steroidal saponin, furcreastatin, from the plant Furcraea foetida. Carbohydrate Research, 323: 57-62.

28. Krasilnikov OV, Capistrano MFP, Yuldasheva LN et al. (1997). Influence of Cys-130 S. aureus alpha-toxin on planar lipid bilayer and erythrocyte membranes. Journal of Membrane Biology, 156: 157-172.

29. Mueller P, Rudin DO, Tien HT et al. (1963). Methods for the formation of single bimolecular lipid membranes in aqueous solution. Journal of Physical Chemistry, 67: 534-535.

30. Chernitskii EA, Sen'kovich OA \& Kozlova NM (1996). Heterogeneity of pores formed in erythrocyte membrane by lipophilic hemolysins. Biofizika, 41: 1270-1274.

31. Abramov AY, Zamaraeva MV, Hagelgans Al et al. (2001). Influence of plant terpenoids on the permeability of mitochondria and lipid bilayers. Biochimica et Biophysica Acta, 1512: 98-110.

32. Wu J, Lin L \& Chau F (2001). Ultrasonic-assisted extraction of ginseng saponins from ginseng roots and cultured ginseng cells. Ultrasonics Sonochemistry, 8: 347-352.

33. Krasilnikov OV, Muratkhodjaev JN \& Zitzer AO (1992). The mode of action of Vibrio cholerae cytolysin. The influences on both erythrocytes and planar lipid bilayers. Biochimica et Biophysica Acta, 1111: 7-16.

34. Yang D-J, Lu T-J \& Hwang LS (2003). Simultaneous determination of furostanol and spirostanol glycosides in Taiwanese yam (Dioscorea spp) cultivars by high performance liquid chromatography. Journal of Food and Drug Analysis, 11: 271-276.

35. Senkovich OA \& Chernitsky EA (1998). On the size of pores arising in erythrocytes under the action of detergents. Membrane and Cell Biology, 11: 679-689.

36. Rowland RN \& Woodley JF (1980). The stability of liposomes in vitro to $\mathrm{pH}$, bile salts and pancreatic lipase. Biochimica et Biophysica Acta, 620: 400-409.

37. Lasch J (1995). Interaction of detergents with lipid vesicles. Biochimica et Biophysica Acta, 1241: 269-292.

38. Steurer S, Wurglics M, Likussar W et al. (1999). Lack of correlation between surface and interfacial activities of saponins and their hemolytic properties. Pharmazie, 54: 766-767.

39. Nakamura T, Nishikawa M, Inoue K et al. (1980). Phosphatidylcholine liposomes containing cholesterol analogues with side chains of various lengths. Chemistry and Physics of Lipids, 26: 101-110. 